\title{
Stochastic comparisons of lifetimes of series and parallel systems with dependent and heterogeneous components
}

\author{
Arindam Panja ${ }^{1}$, Pradip Kundu ${ }^{2 *}$ and Biswabrata Pradhan ${ }^{1}$ \\ ${ }^{1}$ SQC \& OR Unit, Indian Statistical Institute, Kolkata-700108, India \\ 2 Decision Science and Operations Management, Birla Global University, \\ Bhubaneswar, Odisha-751003, India
}

\begin{abstract}
This work considers stochastic comparisons of lifetimes of series and parallel systems with dependent and heterogeneous components having lifetimes following the proportional odds (PO) model. The joint distribution of component lifetimes is modeled by Archimedean survival copula. We discuss some potential applications of our findings in system reliability and actuarial science.
\end{abstract}

Keywords: Archimedean copula; Majorization; Proportional odds model; Stochastic order.

\section{Introduction}

Suppose $X_{1}, X_{2}, \ldots, X_{n}$ are the random variables denoting the lifetimes of the components of a system with $n$ components. Then the system lifetime is a function of $X_{1}, X_{2}, \ldots, X_{n}$. Let $X_{k: n}, k=1,2, \ldots, n$ denote the $k$ th order statistic corresponding to the random variables $X_{1}, X_{2}, \ldots, X_{n}$. Then the smallest and the largest order statistics $X_{1: n}$ and $X_{n: n}$, respectively, represent the lifetimes of the series and the parallel systems. There have been a number of works on stochastic comparisons of system lifetimes where component lifetimes follow different family of distributions [1, 6, 17, 8, 9, 10, 11, 16, 21. However, most of the works have considered mutual independence among the concerned random variables. Recently, Fang et al. [9], Li and Fang [16] and $\mathrm{Li}$ and $\mathrm{Li}$ [17] have considered stochastic comparison of system lifetimes with dependent and heterogeneous component lifetimes following the proportional hazard rate (PHR) model.

The proportional odds (PO) model, as introduced by Bennet [2] and later discussed by Kirmani and Gupta [12] is a very important model in reliability theory and survival analysis. Let

${ }^{*}$ Corresponding author, e-mail: kundu.maths@gmail.com

†Email Address: arindampnj@gmail.com (Arindam Panja), bis@isical.ac.in (Biswabrata Pradhan) 
$X$ and $Y$ be two random variables with distribution functions $F_{X}(\cdot), F_{Y}(\cdot)$, survival functions $\bar{F}_{X}(\cdot), \bar{F}_{Y}(\cdot)$ and hazard rate functions $r_{X}(\cdot), r_{Y}(\cdot)$ respectively. Let the odds functions of $X$ and $Y$ be defined by $\tau_{X}(t)=\bar{F}_{X}(t) / F_{X}(t)$ and $\tau_{Y}(t)=\bar{F}_{Y}(t) / F_{Y}(t)$, respectively. If the random variable $X$ represents the lifetime of a component, then the odds function $\tau_{X}(t)$ represents the odds of that component functioning beyond time $t$. The random variables $X$ and $Y$ are said to satisfy PO model if $\tau_{Y}(t)=\alpha \tau_{X}(t)$ for all admissible $t$, where $\alpha$ is a proportionality constant known as proportional odds ratio. Then the survival functions of $X$ and $Y$ are related as

$$
\bar{F}_{Y}(t)=\frac{\alpha \bar{F}_{X}(t)}{1-\bar{\alpha} \bar{F}_{X}(t)},
$$

where $\bar{\alpha}=1-\alpha$. We will say that the random variable $Y$ is following the PO model with baseline survival function $\bar{F}_{X}(\cdot)$ and parameter (proportionality constant) $\alpha$. For easy interpretation, we can think of $X$ as the lifetime of a member of control group, and $Y$ as that of a member of treatment group. For two random variables satisfying the PO model, the ratio of hazard rates converges to unity as time tends to infinity, which is in contrast to the PHR model where this ratio remains constant with time. The convergence property of hazard functions makes the PO model reasonable in many practical applications as discussed in [2, 12, 22]. For more applications of PO model one may refer to [4, 25]. Also, the model (1), with $0<\alpha<\infty$, provides us a method of generating more flexible new family of distributions by introducing the parameter $\alpha$ to an existing family of distributions [18]. The family of distributions so obtained is known as Marshall-Olkin family of distributions [5, 18. Thus, model (1) has implications both in terms of the PO model and in generating new family of flexible distributions, which makes it worth investigating.

Let $\mathbf{X}=\left(X_{1}, X_{2}, \ldots, X_{n}\right)$ be a random vector with joint distribution function $F(\cdot)$ and joint survival function $\bar{F}(\cdot)$. Also let the distribution function and the survival function of $X_{i}$ are $F_{i}(\cdot)$ and $\bar{F}_{i}(\cdot)$ respectively for $i=1,2, \ldots, n$. The joint distribution of $X_{1}, X_{2}, \ldots, X_{n}$ can be represented by a copula model. If there exist $K:[0,1]^{n} \mapsto[0,1]$ and $\bar{K}:[0,1]^{n} \mapsto[0,1]$ such that $F\left(x_{1}, \ldots, x_{n}\right)=K\left(F_{1}\left(x_{1}\right), \ldots, F_{n}\left(x_{n}\right)\right)$ and $\bar{F}\left(x_{1}, \ldots, x_{n}\right)=\bar{K}\left(\bar{F}_{1}\left(x_{1}\right), \ldots, \bar{F}_{n}\left(x_{n}\right)\right)$ for all $x_{i}, i \in I_{n}$, then $K$ and $\bar{K}$ are called the copula and survival copula of $X$, respectively. If $\varphi:[0,+\infty) \mapsto[0,1]$ with $\varphi(0)=1$ and $\lim _{t \rightarrow+\infty} \varphi(t)=0$ is $(n-2)$ th differentiable, then $K_{\varphi}\left(u_{1}, \ldots, u_{n}\right)=\varphi\left(\varphi^{-1}\left(u_{1}\right)+\ldots+\varphi^{-1}\left(u_{n}\right)\right)=\varphi\left(\sum_{i=1}^{n} \phi\left(u_{i}\right)\right)$ for all $u_{i} \in(0,1], i \in I_{n}$ is called an Archimedean survival copula with generator $\varphi$ provided $(-1)^{k} \varphi^{(k)}(t) \geq 0, k=0,1, \ldots, n-2$ and $(-1)^{n-2} \varphi^{(n-2)}(t)$ is decreasing and convex for all $t \geq 0$. Here $\phi=\varphi^{-1}$ is the right continuous inverse of $\varphi$ so that $\phi(u)=\varphi^{-1}(u)=\sup \{t \in \mathbb{R}: \varphi(t)>u\}$. Navarro and Spizzichino [21] have derived usual stochastic ordering for lifetimes of series and parallel systems having component lifetimes sharing a common copula, with the idea of mean reliability function associated with the common copula. Li and Fang [16] investigated stochastic order between two 
samples of dependent random variables following PHR model and having Archimedean survival copula. Fang et al. [9] derived some stochastic ordering results for minimum as well as for maximum of samples equipped with Archimedean survival copulas and following PHR model and proportional reversed hazard rate $(\mathrm{PRH})$ model, respectively. Li and Li [17] investigated hazard rate order on minimums of sample following PHR model, and reversed hazard rate order on maximums of sample following PRH model, where both the samples coupled with Archimedean survival copula.

In case of PO model, some authors, e.g. Kundu and Nanda [14], Kundu et al. [15], Nanda and Das [20] have investigated stochastic comparison of systems with independent components. However, to the best of our knowledge, no research work has been done on stochastic comparison of system lifetimes with dependent and heterogeneous component lifetimes following PO model. In this work, we investigate stochastic comparisons of lifetimes of series and parallel systems with dependent and heterogenous components having lifetimes following the PO model. The joint distribution of component lifetimes is modeled by Archemedian survival copula. It is shown that the usual stochastic ordering and hazard rate ordering hold for series systems under certain conditions whereas for parallel system stochastic ordering and reversed hazard rate ordering hold.

The organization of the paper is as follows. Section 2 recalls some definitions of majorization, stochastic orders, and some lemmas used in the sequel. In Section 3, we investigate stochastic comparisons between series systems of dependent and heterogenous components having lifetimes following the PO model and coupled by Archimedean survival copulas. Section 4 investigates the same in case of parallel systems. Section 5 presents some potential applications of the proposed results.

\section{Preliminaries}

Given a vector $\boldsymbol{x}=\left(x_{1}, x_{2}, \ldots, x_{n}\right) \in \mathbb{R}^{n}$, denote $x_{(1)} \leq x_{(2)} \leq \ldots \leq x_{(n)}$ as increasing arrangement of $x_{1}, x_{2}, \ldots, x_{n}$.

Definition 2.1 Let $\boldsymbol{x}=\left(x_{1}, x_{2}, \ldots, x_{n}\right)$ and $\boldsymbol{y}=\left(y_{1}, y_{2}, \ldots, y_{n}\right)$ in $\mathbb{R}^{n}$ be any two vectors.

(i) The vector $\boldsymbol{x}$ is said to majorize the vector $\boldsymbol{y}$, i.e., $\boldsymbol{x}$ is larger than $\boldsymbol{y}$ in majorization order (denoted as $\boldsymbol{x} \stackrel{m}{\succeq} \boldsymbol{y}$ ) if (cf. [19])

$$
\sum_{i=1}^{j} x_{(i)} \leq \sum_{i=1}^{j} y_{(i)}, \text { for all } j=1,2, \ldots, n-1, \text { and } \sum_{i=1}^{n} x_{(i)}=\sum_{i=1}^{n} y_{(i)}
$$


(ii) The vector $\boldsymbol{x}$ is said to weakly supermajorize the vector $\boldsymbol{y}$, denoted as $\boldsymbol{x} \succeq \boldsymbol{w}$ if (cf. [19])

$$
\sum_{i=1}^{j} x_{(i)} \leq \sum_{i=1}^{j} y_{(i)}, \text { for all } j=1,2, \ldots, n \text {. }
$$

(iii) The vector $\boldsymbol{x}$ is said to be p-larger than the vector $\boldsymbol{y}$ (denoted as $\boldsymbol{x} \succeq \boldsymbol{p}$ ) if (cf. [3])

$$
\prod_{i=1}^{j} x_{(i)} \leq \prod_{i=1}^{j} y_{(i)}, \text { for all } j=1,2, \ldots, n \text {. }
$$

It can be seen that

$$
\mathrm{x} \stackrel{m}{\succeq} \mathrm{y} \Rightarrow \mathrm{x} \stackrel{w}{\succeq} \mathbf{y} \Rightarrow \mathrm{x} \succeq \stackrel{p}{\underline{y}}
$$

Definition 2.2 [23] Let $X$ and $Y$ be nonnegative absolutely continuous random variables with cumulative distribution functions $F_{X}(\cdot), F_{Y}(\cdot)$, survival functions $\bar{F}_{X}(\cdot), \bar{F}_{Y}(\cdot)$, hazard (failure) rate functions $r_{X}(\cdot), r_{Y}(\cdot)$, and the reversed hazard rate functions $\tilde{r}_{X}(\cdot)$ and $\tilde{r}_{Y}(\cdot)$, respectively. Then $X$ is said to be smaller than $Y$ in the

(i) usual stochastic order (denoted as $X \leq_{s t} Y$ ) if $\bar{F}_{X}(t) \leq \bar{F}_{Y}(t)$ for all $t$;

(ii) hazard rate order (denoted as $X \leq_{h r} Y$ ) if $\bar{F}_{Y}(t) / \bar{F}_{X}(t)$ is increasing in $t \geq 0$, or equivalently if $r_{X}(t) \geq r_{Y}(t)$ for all $t \geq 0$;

(iii) reversed hazard rate order (denoted as $X \leq_{r h r} Y$ ) if $F_{Y}(t) / F_{X}(t)$ is increasing in $t>0$, or equivalently if $\tilde{r}_{X}(t) \leq \tilde{r}_{Y}(t)$ for all $t>0$.

Lemma 2.1 [19] Let $I \subseteq \mathbb{R}$ be an open interval and let $\zeta: I^{n} \rightarrow \mathbb{R}$ be continuously differentiable. Necessary and sufficient conditions for $\zeta$ to be Schur-convex (resp. Schur-concave) on $I^{n}$ are that $\zeta$ is symmetric on $I^{n}$, and for all $i \neq j$,

$$
\left(u_{i}-u_{j}\right)\left(\zeta_{(i)}(\boldsymbol{u})-\zeta_{(j)}(\boldsymbol{u})\right) \geq(\text { resp. } \leq) 0 \text { for all } \boldsymbol{u}=\left(u_{1}, u_{2}, \ldots, u_{n}\right) \in I^{n}
$$

where $\zeta_{(k)}(\boldsymbol{u})=\partial \zeta(\boldsymbol{u}) / \partial u_{k}$

Lemma 2.2[19] Let $\mathcal{A} \subseteq \mathbb{R}^{n}$, and $\zeta: \mathcal{A} \rightarrow \mathbb{R}$ be a function. Then, for $\boldsymbol{x}, \boldsymbol{y} \in \mathcal{A}$,

$$
\boldsymbol{x} \succeq \boldsymbol{w} \Longrightarrow \zeta(\boldsymbol{x}) \geq(\text { resp. } \leq) \zeta(\boldsymbol{y})
$$

if and only if $\zeta$ is both decreasing (resp. increasing) and Schur-convex (resp. Schur-concave) on $\mathcal{A}$. 
Lemma 2.3 [13] Let $\zeta:(0, \infty)^{n} \rightarrow \mathbb{R}$ be a function. Then,

$$
\boldsymbol{x} \stackrel{\mathrm{p}}{\succeq} \Longrightarrow \zeta(\boldsymbol{x}) \geq(\text { resp. } \leq) \zeta(\boldsymbol{y})
$$

if and only if the following two conditions hold:

(i) $\zeta\left(e^{v_{1}}, \ldots, e^{v_{n}}\right)$ is Schur-convex (resp. Schur-concave) in $\left(v_{1}, \ldots, v_{n}\right)$,

(ii) $\zeta\left(e^{v_{1}}, \ldots, e^{v_{n}}\right)$ is decreasing (resp. increasing) in each $v_{i}$, for $i=1, \ldots, n$,

where $v_{i}=\ln x_{i}$, for $i=1, \ldots, n$.

Lemma 2.4 [9] For two n-dimensional Archimedean copulas $K_{\varphi_{1}}$ and $K_{\varphi_{2}}$, if $\phi_{2} \circ \varphi_{1}$ is superadditive, then $K_{\varphi_{1}}(\boldsymbol{u}) \leq K_{\varphi_{2}}(\boldsymbol{u})$ for all $\boldsymbol{u} \in[0,1]^{n}$.

\section{Series systems with dependent and heterogeneous component lifetimes following PO Model}

Here, we consider the comparison of lifetimes of two series systems with heterogeneous and dependent components. We assume that the lifetime vector $X=\left(X_{1}, X_{2}, \ldots, X_{n}\right)$ is a set of dependent random variables coupled with Archimedean survival copula with generator $\varphi$ and following the PO model with baseline survival function $\bar{F}$, denoted as $X \sim P O(\bar{F}, \boldsymbol{\alpha}, \varphi)$, where $\boldsymbol{\alpha}=\left(\alpha_{1}, \alpha_{2}, \ldots, \alpha_{n}\right) \in \mathbb{R}_{+}^{n}$ is the proportional odds ratio vector. The survival function and the hazard rate function of $X_{i}$ are

$$
\bar{F}_{\alpha_{i}}(x)=\frac{\alpha_{i} \bar{F}(x)}{1-\bar{\alpha}_{i} \bar{F}(x)} \text { and } r_{\alpha_{i}}(x)=\frac{r(x)}{1-\bar{\alpha}_{i} \bar{F}(x)} \text {, respectively, }
$$

where $\bar{\alpha}_{i}=1-\alpha_{i}, i=1, \ldots, n$ and $r$ denotes the baseline hazard rate function. The survival functions of $X_{1: n}$ is given by

$$
\bar{F}_{X_{1: n}}(x)=P\left(X_{1: n}>x\right)=P\left(X_{i}>x, i \in I_{n}\right)=\varphi\left(\sum_{i=1}^{n} \phi\left(\bar{F}_{\alpha_{i}}(x)\right)\right)=S_{1}(\bar{F}(x), \boldsymbol{\alpha}, \varphi), \text { say }
$$

where $\phi(u)=\varphi^{-1}(u), u \in(0,1]$.

The hazard rate function of $X_{1: n}$ is obtained as

$$
r_{X_{1: n}}(x)=r(x) \frac{\varphi^{\prime}\left(\sum_{i=1}^{n} \phi\left(\bar{F}_{\alpha_{i}}(x)\right)\right)}{\varphi\left(\sum_{i=1}^{n} \phi\left(\bar{F}_{\alpha_{i}}(x)\right)\right)} \sum_{i=1}^{n} \phi^{\prime}\left(\bar{F}_{\alpha_{i}}(x)\right) \frac{\bar{F}_{\alpha_{i}}(x)}{1-\bar{\alpha}_{i} \bar{F}(x)} .
$$

Lemma 3.1 For any $x \in[0,1], S_{1}(x, \boldsymbol{\alpha}, \varphi)$ is increasing in $\alpha_{i}, i \in I_{n}$. Furthermore $S_{1}$ is Schur-concave with respect to $\boldsymbol{\alpha}$. 
Proof: For $s \in I_{n}$,

$$
\frac{\partial S_{1}}{\partial \alpha_{s}}=\varphi^{\prime}\left(\sum_{i=1}^{n} \phi\left(\frac{\alpha_{i} x}{1-\bar{\alpha}_{i} x}\right)\right) \phi^{\prime}\left(\frac{\alpha_{s} x}{1-\bar{\alpha}_{s} x}\right) \frac{x(1-x)}{\left(1-\bar{\alpha}_{s} x\right)^{2}}
$$

Since both $\varphi(u)$ and $\phi(u)$ are decreasing for all $u \geq 0, \frac{\partial S_{1}}{\partial \alpha_{s}} \geq 0$. As a result $S_{1}(x, \boldsymbol{\alpha}, \varphi)$ is increasing in $\alpha_{i}, i \in I_{n}$ for any $x \in[0,1]$.

For $s \neq t$,

$$
\begin{aligned}
& \left(\alpha_{s}-\alpha_{t}\right)\left(\frac{\partial S_{1}}{\partial \alpha_{s}}-\frac{\partial S_{1}}{\partial \alpha_{t}}\right) \\
= & \left(\alpha_{s}-\alpha_{t}\right) \varphi^{\prime}\left(\sum_{i=1}^{n} \phi\left(\frac{\alpha_{i} x}{1-\bar{\alpha}_{i} x}\right)\right)\left[\phi^{\prime}\left(\frac{\alpha_{s} x}{1-\bar{\alpha}_{s} x}\right) \frac{x(1-x)}{\left(1-\bar{\alpha}_{s} x\right)^{2}}-\phi^{\prime}\left(\frac{\alpha_{t} x}{1-\bar{\alpha}_{t} x}\right) \frac{x(1-x)}{\left(1-\bar{\alpha}_{t} x\right)^{2}}\right] \\
\stackrel{\text { sign }}{=} & \left(\alpha_{s}-\alpha_{t}\right)\left(-\varphi^{\prime}\left(\sum_{i=1}^{n} \phi\left(u_{i}\right)\right)\right)\left[\left(-\phi^{\prime}\left(u_{s}\right)\right) \frac{1}{\left(1-\bar{\alpha}_{s} x\right)^{2}}-\left(-\phi^{\prime}\left(u_{t}\right)\right) \frac{1}{\left(1-\bar{\alpha}_{t} x\right)^{2}}\right],
\end{aligned}
$$

where $u_{i}=\frac{\alpha_{i} x}{1-\bar{\alpha}_{i} x}$ and $\stackrel{\text { 'sign' }}{=}$ means equal in sign. Since both $\varphi$ and $\phi$ are decreasing, and $\phi^{\prime}$ is increasing, it follows from 44 that $\left(\alpha_{s}-\alpha_{t}\right)\left(\frac{\partial S_{1}}{\partial \alpha_{s}}-\frac{\partial S_{1}}{\partial \alpha_{t}}\right) \leq 0$. So from Lemma 2.1, $S_{1}$ is Schur-concave in $\boldsymbol{\alpha}=\left(\alpha_{1}, \alpha_{2}, \ldots, \alpha_{n}\right)$.

Suppose there are two series systems formed out of $n$ statistically dependent and heterogeneous components where the component lifetimes follow the PO model. The joint distribution of lifetimes of components is represented by Archimedean copula. Consider two such series systems with lifetime vectors $X=\left(X_{1}, X_{2}, \ldots, X_{n}\right)$ and $Y=\left(Y_{1}, Y_{2}, \ldots, Y_{n}\right)$ having respective proportionality odds ratio vectors $\boldsymbol{\alpha}=\left(\alpha_{1}, \alpha_{2}, \ldots, \alpha_{n}\right)$ and $\boldsymbol{\beta}=\left(\beta_{1}, \beta_{2}, \ldots, \beta_{n}\right)$, where $\boldsymbol{\alpha}, \boldsymbol{\beta} \in \mathbb{R}_{+}^{n}$. The following theorem compares the lifetimes of these series systems in the sense of usual stochastic order.

Theorem 3.1 Suppose the lifetime vectors $X \sim P O\left(\bar{F}, \boldsymbol{\alpha}, \varphi_{1}\right)$ and $Y \sim P O\left(\bar{F}, \boldsymbol{\beta}, \varphi_{2}\right)$. If $\varphi_{1}$ or $\varphi_{2}$ is log-convex and $\phi_{2} \circ \varphi_{1}$ is superadditive, then

$$
\boldsymbol{\alpha} \succeq \boldsymbol{\beta} \text { implies } X_{1: n} \leq_{s t} Y_{1: n}
$$

Proof: Write $v_{i}=\ln \alpha_{i}, i=1,2, \ldots, n$. Then as per (2),

$$
\bar{F}_{X_{1: n}}(x)=\varphi_{1}\left(\sum_{i=1}^{n} \phi_{1}\left(\frac{e^{v_{i}} \bar{F}(x)}{1-\left(1-e^{v_{i}}\right) \bar{F}(x)}\right)\right)=S_{1}\left(\bar{F}(x),\left(e^{v_{1}}, e^{v_{2}}, \ldots, e^{v_{n}}\right), \varphi_{1}\right)
$$

Here $S_{1}\left(\bar{F}(x),\left(e^{v_{1}}, e^{v_{2}}, \ldots, e^{v_{n}}\right), \varphi_{1}\right)$ is symmetric with respect to $\left(v_{1}, v_{2}, \ldots, v_{n}\right) \in \mathbb{R}^{n}$. Now, for 
$s \in I_{n}$,

$$
\frac{\partial S_{1}}{\partial v_{s}}=\varphi_{1}^{\prime}\left(\sum_{i=1}^{n} \phi_{1}\left(\frac{e^{v_{i}} x}{1-\left(1-e^{v_{i}}\right) x}\right)\right) \phi_{1}^{\prime}\left(\frac{e^{v_{s}} x}{1-\left(1-e^{v_{s}}\right) x}\right) \frac{x(1-x) e^{v_{s}}}{\left(1-\left(1-e^{v_{s}}\right) x\right)^{2}}
$$

so that $S_{1}\left(x,\left(e^{v_{1}}, e^{v_{2}}, \ldots, e^{v_{n}}\right), \varphi_{1}\right)$ is increasing in each $v_{i}, i=1,2, \ldots, n$ for any $x \in[0,1]$.

Now, for $s \neq t$,

$$
\begin{aligned}
& \left(v_{s}-v_{t}\right)\left(\frac{\partial S_{1}}{\partial v_{s}}-\frac{\partial S_{1}}{\partial v_{t}}\right) \\
= & \left(v_{s}-v_{t}\right)\left(-\varphi_{1}^{\prime}\left(\sum_{i=1}^{n} \phi_{1}\left(\frac{e^{v_{i}} x}{1-\left(1-e^{v_{i}}\right) x}\right)\right)\right)\left[\left(-\phi_{1}^{\prime}\left(\frac{e^{v_{s}} x}{1-\left(1-e^{v_{s}}\right) x}\right)\right) \frac{x e^{v_{s}}}{\left(1-\left(1-e^{v_{s}}\right) x\right)^{2}}\right. \\
& \left.-\left(-\phi_{1}^{\prime}\left(\frac{e^{v_{t}} x}{1-\left(1-e^{v_{t}}\right) x}\right)\right) \frac{x e^{v_{t}}}{\left(1-\left(1-e^{v_{t}}\right) x\right)^{2}}\right] \\
\underline{\text { sign }} & \left(v_{s}-v_{t}\right)\left[\left(-\frac{\varphi_{1}\left(\phi_{1}\left(u_{s}\right)\right)}{\varphi_{1}^{\prime}\left(\phi_{1}\left(u_{s}\right)\right)}\right) \frac{1}{1-\left(1-e^{v_{s}}\right) x}-\left(-\frac{\varphi_{1}\left(\phi_{1}\left(u_{t}\right)\right)}{\varphi_{1}^{\prime}\left(\phi_{1}\left(u_{t}\right)\right)}\right) \frac{1}{1-\left(1-e^{v_{t}}\right) x}\right],
\end{aligned}
$$

where $u_{s}=\frac{e^{v_{s}} x}{1-\left(1-e^{v_{s}}\right) x}$. If $\varphi_{1}$ is log-convex, from 5 it follows that $\left(v_{s}-v_{t}\right)\left(\frac{\partial S_{1}}{\partial v_{s}}-\frac{\partial S_{1}}{\partial v_{t}}\right) \leq 0$. Hence from Lemma 2.1, $S_{1}\left(x,\left(e^{v_{1}}, e^{v_{2}}, \ldots, e^{v_{n}}\right), \varphi_{1}\right)$ is Schur-concave in $\left(v_{1}, v_{2}, \ldots, v_{n}\right)$ if $\varphi_{1}$ is log-convex. Then from Lemma 2.3 , we have

$$
\boldsymbol{\alpha} \succeq \boldsymbol{\beta} \text { implies } S_{1}\left(\bar{F}(x), \boldsymbol{\alpha}, \varphi_{1}\right) \leq S_{1}\left(\bar{F}(x), \boldsymbol{\beta}, \varphi_{1}\right) .
$$

Since $\phi_{2} \circ \varphi_{1}$ is superadditive, from Lemma 2.4, we have

$$
S_{1}\left(\bar{F}(x), \boldsymbol{\beta}, \varphi_{1}\right) \leq S_{1}\left(\bar{F}(x), \boldsymbol{\beta}, \varphi_{2}\right)
$$

Thus combining (6) and (7) we get $S_{1}\left(\bar{F}(x), \boldsymbol{\alpha}, \varphi_{1}\right) \leq S_{1}\left(\bar{F}(x), \boldsymbol{\beta}, \varphi_{2}\right)$, that is $X_{1: n} \leq_{s t} Y_{1: n}$. Now suppose $\varphi_{2}$ is log-convex, then $S_{1}\left(\bar{F}(x), \boldsymbol{\alpha}, \varphi_{2}\right) \leq S_{1}\left(\bar{F}(x), \boldsymbol{\beta}, \varphi_{2}\right)$. Since $\phi_{2} \circ \varphi_{1}$ is superadditive, we have $S_{1}\left(\bar{F}(x), \boldsymbol{\alpha}, \varphi_{1}\right) \leq S_{1}\left(\bar{F}(x), \boldsymbol{\alpha}, \varphi_{2}\right)$. So combining we get $X_{1: n} \leq_{s t} Y_{1: n}$.

Corollary 3.1 Suppose the lifetime vectors $X \sim P O(\bar{F}, \boldsymbol{\alpha}, \varphi)$ and $Y \sim P O(\bar{F}, \boldsymbol{\beta}, \varphi)$. If $\varphi$ is log-convex, then

$$
\boldsymbol{\alpha} \succeq \boldsymbol{p} \text { implies } X_{1: n} \leq_{s t} Y_{1: n}
$$

The following counterexample shows that one may not get the the ordering result in Theorem 3.1 if the sufficient conditions on the generator functions are dropped.

Counterexample 3.1 Consider two series systems, each comprising of three dependent and heterogeneous components with respective survival functions $\bar{F}_{X_{1: 3}}(x)=S_{1}\left(\bar{F}(x), \boldsymbol{\alpha}, \varphi_{1}\right)$ and $\bar{F}_{Y_{1: 3}}(x)=S_{1}\left(\bar{F}(x), \boldsymbol{\beta}, \varphi_{2}\right)$ with $\bar{F}(x)=e^{-(x)^{1.5}}, x \geq 0, \boldsymbol{\alpha}=(2,3,5.5), \boldsymbol{\beta}=(2.5,3.5,3.8)$ so 


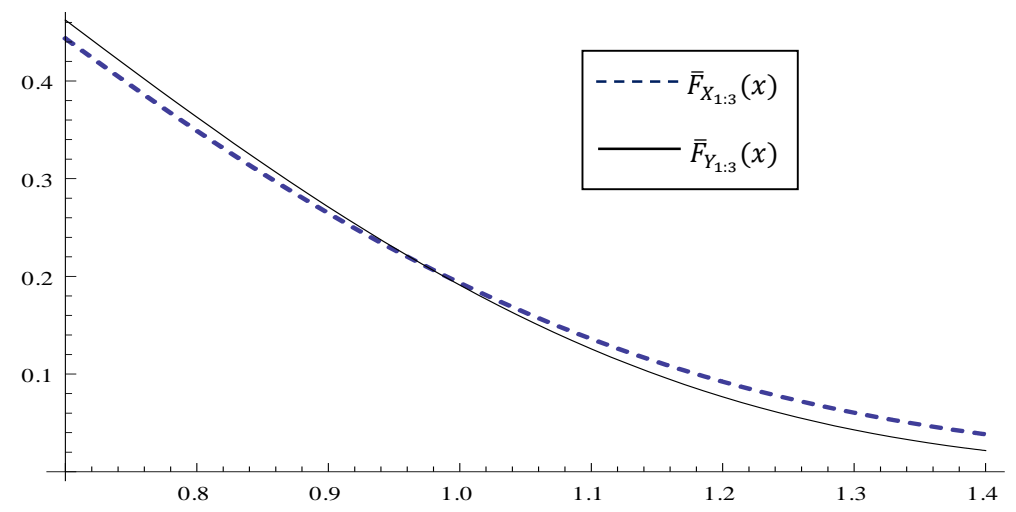

Figure 1: Plots of $\bar{F}_{X_{1: 3}}(x)$ and $\bar{F}_{Y_{1: 3}}(x)$ when $\phi_{2} \circ \varphi_{1}$ is not super additive, and $\varphi_{1}$ and $\varphi_{2}$ are not log-convex.

that $\boldsymbol{\alpha} \succeq \boldsymbol{p} \boldsymbol{\beta}$. First we take $\varphi_{1}(x)=\left(2 /\left(1+e^{x}\right)\right)^{1 / \theta}, \theta=0.9$ and $\varphi_{2}(x)=e^{1-(1+x)^{1 / \eta}}$ with $\eta=0.3$ so that $\phi_{2} \circ \varphi_{1}$ is not super additive, and $\varphi_{1}$ and $\varphi_{2}$ are not log-convex. We depict $\bar{F}_{X_{1: 3}}(x)$ and $\bar{F}_{Y_{1: 3}}(x)$ in Figure 1 for some finite range of $x$. From this figure we observe that the stochastic ordering result in Theorem 3.1 is not attained.

Since $p$-larger order is weaker than weakly supermajorization order, the following theorem shows that we can get the ordering result in Theorem 3.1 under weakly supermajorization order with fewer condition.

Theorem 3.2 Suppose the lifetime vectors $X \sim P O\left(\bar{F}, \boldsymbol{\alpha}, \varphi_{1}\right)$ and $Y \sim P O\left(\bar{F}, \boldsymbol{\beta}, \varphi_{2}\right)$. If $\phi_{2} \circ \varphi_{1}$ is superadditive, then

$$
\boldsymbol{\alpha} \succeq \boldsymbol{\beta} \text { implies } X_{1: n} \leq_{s t} Y_{1: n}
$$

Proof: From Lemma 3.1 and Lemma 2.2, we have

$$
\boldsymbol{\alpha} \succeq \boldsymbol{\beta} \text { implies } S_{1}\left(\bar{F}(x), \boldsymbol{\alpha}, \varphi_{1}\right) \leq S_{1}\left(\bar{F}(x), \boldsymbol{\beta}, \varphi_{1}\right)
$$

Since $\phi_{2} \circ \varphi_{1}$ is superadditive, so from Lemma 2.4 , we have, $S_{1}\left(\bar{F}(x), \boldsymbol{\beta}, \varphi_{1}\right) \leq S_{1}\left(\bar{F}(x), \boldsymbol{\beta}, \varphi_{2}\right)$. Combining the above results we have $S_{1}\left(\bar{F}(x), \boldsymbol{\alpha}, \varphi_{1}\right) \leq S_{1}\left(\bar{F}(x), \boldsymbol{\beta}, \varphi_{2}\right)$. That is $X_{1: n} \leq_{s t} Y_{1: n} . \square$

Remark 3.1 It is to be noted that super-additive assumption of $\phi_{2} \circ \varphi_{1}$ is satisfied by many members of Archimedean survival copulas. For example, Archimedean survival copula with generators (i) $\varphi_{1}(t)=e^{1-(1+t)^{\frac{1}{\theta}}}$ and $\varphi_{2}(t)=\frac{\theta}{\log \left(e^{\theta}+t\right)}$, where $0<\theta \leq 1$, (ii) $\varphi_{1}(t)=\frac{\theta}{\log \left(e^{\theta}+t\right)}$ and $\varphi_{2}(t)=\log (e+t)^{-1 / \theta}$, where $\theta>1$ and (iii) $\varphi_{1}(t)=e^{1-(1+t)^{\frac{1}{\theta_{1}}}}$ and $\varphi_{2}(t)=e^{1-(1+t)^{\frac{1}{\theta_{2}}}}$, where $\theta_{2} \geq \theta_{1} \geq 1$, satisfy super-additivity. 
Corollary 3.2 Suppose the lifetime vectors $X \sim P O(\bar{F}, \boldsymbol{\alpha}, \varphi)$ and $Y \sim P O(\bar{F}, \boldsymbol{\beta}, \varphi)$. Then

$$
\boldsymbol{\alpha} \succeq \boldsymbol{\beta} \text { implies } X_{1: n} \leq_{s t} Y_{1: n}
$$

Lemma 3.2 $I_{1}(\boldsymbol{u})=\frac{\varphi^{\prime}\left(\sum_{i=1}^{n} u_{i}\right)}{\varphi\left(\sum_{i=1}^{n} u_{i}\right)} \sum_{i=1}^{n} \frac{\varphi\left(u_{i}\right)}{\varphi^{\prime}\left(u_{i}\right)}\left(1-\varphi\left(u_{i}\right)\right)$ is increasing in $u_{s}, s \in I_{n}$ and Schurconvex with respect to $\boldsymbol{u}=\left(u_{1}, \ldots, u_{n}\right)$ if $\varphi$ is log-concave and $\frac{\varphi(1-\varphi)}{\varphi^{\prime}}$ is decreasing and concave.

Proof: Here $I_{1}(\mathbf{u})$ is symmetric in $\mathbf{u}$. For $s \in I_{n}$,

$$
\frac{\partial I_{1}(\mathbf{u})}{\partial u_{s}}=\frac{\partial}{\partial u_{s}}\left(\frac{\varphi^{\prime}\left(\sum_{i=1}^{n} u_{i}\right)}{\varphi\left(\sum_{i=1}^{n} u_{i}\right)}\right) \sum_{i=1}^{n} \frac{\varphi\left(u_{i}\right)}{\varphi^{\prime}\left(u_{i}\right)}\left(1-\varphi\left(u_{i}\right)\right)+\frac{\varphi^{\prime}\left(\sum_{i=1}^{n} u_{i}\right)}{\varphi\left(\sum_{i=1}^{n} u_{i}\right)} \frac{\partial}{\partial u_{s}}\left(\frac{\varphi\left(u_{s}\right)}{\varphi^{\prime}\left(u_{s}\right)}\left(1-\varphi\left(u_{s}\right)\right)\right) .
$$

Since $\varphi$ is log-concave, $\frac{\partial}{\partial u_{s}}\left(\frac{\varphi^{\prime}\left(\sum_{i=1}^{n} u_{i}\right)}{\varphi\left(\sum_{i=1}^{n} u_{i}\right)}\right) \leq 0$. As $\varphi(1-\varphi) / \varphi^{\prime}$ is decreasing, $\frac{\partial}{\partial u_{s}}\left(\frac{\varphi\left(u_{s}\right)}{\varphi^{\prime}\left(u_{s}\right)}\left(1-\varphi\left(u_{s}\right)\right)\right) \leq$ 0 . Then using the fact that $\varphi$ is deceasing, we have $\frac{\partial I_{1}(\mathbf{u})}{\partial u_{s}} \geq 0$. So $I_{1}(\mathbf{u})$ is increasing in $u_{s}$ for any $s \in I_{n}$. For $s, t \in I_{n}$ with $s \neq t$,

$$
\frac{\partial}{\partial u_{s}}\left(\frac{\varphi^{\prime}\left(\sum_{i=1}^{n} u_{i}\right)}{\varphi\left(\sum_{i=1}^{n} u_{i}\right)}\right)=\frac{\partial}{\partial u_{t}}\left(\frac{\varphi^{\prime}\left(\sum_{i=1}^{n} u_{i}\right)}{\varphi\left(\sum_{i=1}^{n} u_{i}\right)}\right)
$$

Then

$$
\begin{aligned}
& \left(u_{s}-u_{t}\right)\left(\frac{\partial I_{1}(\mathbf{u})}{\partial u_{s}}-\frac{\partial I_{1}(\mathbf{u})}{\partial u_{t}}\right) \\
= & \left(u_{s}-u_{t}\right) \frac{\varphi^{\prime}\left(\sum_{i=1}^{n} u_{i}\right)}{\varphi\left(\sum_{i=1}^{n} u_{i}\right)}\left[\frac{\partial}{\partial u_{s}}\left(\frac{\varphi\left(u_{s}\right)}{\varphi^{\prime}\left(u_{s}\right)}\left(1-\varphi\left(u_{s}\right)\right)\right)-\frac{\partial}{\partial u_{t}}\left(\frac{\varphi\left(u_{t}\right)}{\varphi^{\prime}\left(u_{t}\right)}\left(1-\varphi\left(u_{t}\right)\right)\right)\right] \geq 0
\end{aligned}
$$

where the inequality follows from the fact that $\frac{\varphi(1-\varphi)}{\varphi^{\prime}}$ is concave. So from lemma 2.1 , $I_{1}(\mathbf{u})$ is Schur-convex with respect to $\mathbf{u}$.

Next we show hazard rate ordering of two series systems formed out of $n$ statistically dependent and heterogeneous components having lifetimes following PO model.

Theorem 3.3 Suppose the lifetime vectors $X \sim P O(\bar{F}, \boldsymbol{\alpha}, \varphi)$ and $Y \sim P O(\bar{F}, \boldsymbol{\beta}, \varphi)$. If $\varphi$ is log-concave and $\frac{\varphi(1-\varphi)}{\varphi^{\prime}}$ is decreasing and concave (or convex), then

$$
\boldsymbol{\alpha} \succeq \boldsymbol{\beta} \text { implies } X_{1: n} \leq_{h r} Y_{1: n}
$$


Proof: From (3), we have

$$
\begin{aligned}
r_{X_{1: n}}(x) & =r(x) \frac{\varphi^{\prime}\left(\sum_{i=1}^{n} \phi\left(\bar{F}_{\alpha_{i}}(x)\right)\right)}{\varphi\left(\sum_{i=1}^{n} \phi\left(\bar{F}_{\alpha_{i}}(x)\right)\right)} \sum_{i=1}^{n} \phi^{\prime}\left(\bar{F}_{\alpha_{i}}(x)\right) \frac{\bar{F}_{\alpha_{i}}(x)}{1-\bar{\alpha}_{i} \bar{F}(x)} \\
& =\frac{r(x)}{F(x)} \frac{\varphi^{\prime}\left(\sum_{i=1}^{n} \phi\left(\bar{F}_{\alpha_{i}}(x)\right)\right)}{\varphi\left(\sum_{i=1}^{n} \phi\left(\bar{F}_{\alpha_{i}}(x)\right)\right)} \sum_{i=1}^{n} \frac{\bar{F}_{\alpha_{i}}(x)}{\varphi^{\prime}\left(\phi\left(\bar{F}_{\alpha_{i}}(x)\right)\right)} \frac{F(x)}{1-\bar{\alpha}_{i} \bar{F}(x)} \\
& =\frac{r(x)}{F(x)} I_{1}\left(\phi\left(\bar{F}_{\alpha_{1}}(x)\right), \ldots, \phi\left(\bar{F}_{\alpha_{n}}(x)\right)\right),
\end{aligned}
$$

where

$I_{1}\left(\phi\left(\bar{F}_{\alpha_{1}}(x)\right), \ldots, \phi\left(\bar{F}_{\alpha_{n}}(x)\right)\right)=\frac{\varphi^{\prime}\left(\sum_{i=1}^{n} \phi\left(\bar{F}_{\alpha_{i}}(x)\right)\right)}{\varphi\left(\sum_{i=1}^{n} \phi\left(\bar{F}_{\alpha_{i}}(x)\right)\right)} \sum_{i=1}^{n} \frac{\varphi\left(\phi\left(\bar{F}_{\alpha_{i}}(x)\right)\right)}{\varphi^{\prime}\left(\phi\left(\bar{F}_{\alpha_{i}}(x)\right)\right)}\left(1-\varphi\left(\phi\left(\bar{F}_{\alpha_{i}}(x)\right)\right)\right)$.

It is easy to check that $\phi\left(\bar{F}_{\alpha_{i}}(x)\right)$ is decreasing and convex in $\alpha_{i}$. From Theorem A.2 (Chapter 5) of Marshall et al. [19], $\boldsymbol{\alpha} \succeq \boldsymbol{w}$ implies $\left(\phi\left(\bar{F}_{\alpha_{1}}(x)\right), \ldots, \phi\left(\bar{F}_{\alpha_{n}}(x)\right)\right) \succeq_{w}\left(\phi\left(\bar{F}_{\beta_{1}}(x)\right), \ldots, \phi\left(\bar{F}_{\beta_{n}}(x)\right)\right)$. From Lemma 3.2, $I_{1}(\mathbf{u})$ is increasing in $u_{i}$ for $i \in I_{n}$ and Schur-convex with respect to $\mathbf{u}$ whenever $\varphi$ is $\log$-concave and $\frac{\varphi(1-\varphi)}{\varphi^{\prime}}$ is decreasing and concave. Then from Theorem A.8 (Chapter 3) of Marshall et al. [19], we get

$$
I_{1}\left(\phi\left(\bar{F}_{\alpha_{1}}(x)\right), \ldots, \phi\left(\bar{F}_{\alpha_{n}}(x)\right)\right) \geq I_{1}\left(\phi\left(\bar{F}_{\beta_{1}}(x)\right), \ldots, \phi\left(\bar{F}_{\beta_{n}}(x)\right)\right)
$$

which implies $r_{X_{1: n}}(x) \geq r_{Y_{1: n}}(x)$, that is $X_{1: n} \leq{ }_{h r} Y_{1: n}$.

Next we prove the theorem when $\frac{\varphi(1-\varphi)}{\varphi^{\prime}}$ is convex. Let $z_{i}=\phi\left(\bar{F}_{\alpha_{i}}(x)\right)$. Then the hazard rate function is given by

$$
r_{X_{1: n}}(x)=\frac{r(x)}{F(x)} \frac{\varphi^{\prime}\left(\sum_{i=1}^{n} z_{i}\right)}{\varphi\left(\sum_{i=1}^{n} z_{i}\right)} \sum_{i=1}^{n} \frac{\varphi\left(z_{i}\right)}{\varphi^{\prime}\left(z_{i}\right)}\left(1-\varphi\left(z_{i}\right)\right) .
$$

Now, for $s \in I_{n}$,

$$
\begin{aligned}
\frac{r_{X_{1: n}}(x)}{\partial \alpha_{s}}= & \frac{r(x)}{F(x)}\left[\frac{\partial}{\partial z_{s}}\left(\frac{\varphi^{\prime}\left(\sum_{i=1}^{n} z_{i}\right)}{\varphi\left(\sum_{i=1}^{n} z_{i}\right)}\right) \frac{\partial z_{s}}{\partial \alpha_{s}} \sum_{i=1}^{n} \frac{\varphi\left(z_{i}\right)\left(1-\varphi\left(z_{i}\right)\right)}{\varphi^{\prime}\left(z_{i}\right)}+\right. \\
& \left.\frac{\varphi^{\prime}\left(\sum_{i=1}^{n} z_{i}\right)}{\varphi\left(\sum_{i=1}^{n} z_{i}\right)} \frac{\partial}{\partial z_{s}}\left(\frac{\varphi\left(z_{s}\right)\left(1-\varphi\left(z_{s}\right)\right)}{\varphi^{\prime}\left(z_{s}\right)}\right) \frac{\partial z_{s}}{\partial \alpha_{s}}\right]
\end{aligned}
$$

Note that $z_{s}$ is decreasing in $\alpha_{s}$ and $\frac{\partial z_{s}}{\partial \alpha_{s}}$ is increasing in $\alpha_{s}$. Since $\varphi$ is log-concave and $\frac{\varphi(1-\varphi)}{\varphi^{\prime}}$ is decreasing, we have $\frac{r_{X_{1: n}}(x)}{\partial \alpha_{s}} \leq 0$. Again

$$
\frac{\partial}{\partial z_{s}}\left(\frac{\varphi^{\prime}\left(\sum_{i=1}^{n} z_{i}\right)}{\varphi\left(\sum_{i=1}^{n} z_{i}\right)}\right)=\frac{\partial}{\partial z_{t}}\left(\frac{\varphi^{\prime}\left(\sum_{i=1}^{n} z_{i}\right)}{\varphi\left(\sum_{i=1}^{n} z_{i}\right)}\right), \text { for } s \neq t
$$


For $s \neq t$,

$$
\begin{array}{ll} 
& \left(\alpha_{s}-\alpha_{t}\right)\left(\frac{r_{X_{1: n}}}{\partial \alpha_{s}}-\frac{r_{X_{1: n}}}{\partial \alpha_{t}}\right) \\
\stackrel{\text { sign }}{=}\left(\alpha_{s}-\alpha_{t}\right)\left(\frac{\partial z_{s}}{\partial \alpha_{s}}-\frac{\partial z_{t}}{\partial \alpha_{t}}\right)+\left(\alpha_{s}-\alpha_{t}\right) \frac{\varphi^{\prime}\left(\sum_{i=1}^{n} z_{i}\right)}{\varphi\left(\sum_{i=1}^{n} z_{i}\right)} \times \\
& {\left[\left(-\frac{\partial}{\partial z_{s}}\left(\frac{\varphi\left(z_{s}\right)\left(1-\varphi\left(z_{s}\right)\right)}{\varphi^{\prime}\left(z_{s}\right)}\right)\right)\left(-\frac{\partial z_{s}}{\partial \alpha_{s}}\right)-\left(-\frac{\partial}{\partial z_{t}}\left(\frac{\varphi\left(z_{t}\right)\left(1-\varphi\left(z_{t}\right)\right)}{\varphi^{\prime}\left(z_{t}\right)}\right)\right)\left(-\frac{\partial z_{t}}{\partial \alpha_{t}}\right)\right]} \\
\leq & 0,
\end{array}
$$

whenever $\frac{\varphi(1-\varphi)}{\varphi^{\prime}}$ is convex in addition to the log-concave $\varphi$ and decreasing $\frac{\varphi(1-\varphi)}{\varphi^{\prime}}$. Thus we have $r_{X_{1: n}}(x)$ is decreasing in $\alpha_{i}, i \in I_{n}$ and Schur-convex in $\boldsymbol{\alpha}=\left(\alpha_{1}, \alpha_{2}, \ldots, \alpha_{n}\right)$. Then from Lemma 2.2, we have

$$
\boldsymbol{\alpha} \succeq \boldsymbol{\beta} \text { implies } r_{X_{1: n}}(x) \geq r_{X_{1: n}}(x)
$$

Hence the theorem follows.

Corollary 3.3 Suppose the lifetime vectors $X \sim P O(\bar{F}, \boldsymbol{\alpha}, \varphi)$ and $Y \sim P O(\bar{F}, \alpha \mathbf{1}, \varphi)$. Then, $X_{1: n} \leq_{h r} Y_{1: n}$ if $\alpha \geq \frac{1}{n} \sum_{i=1}^{n} \alpha_{i}, \varphi$ is log-concave and $\frac{\varphi(1-\varphi)}{\varphi^{\prime}}$ is decreasing and concave (or convex). This follows from the Theorem 3.3 and using the fact that $\left(\alpha_{1}, \alpha_{2}, \ldots, \alpha_{n}\right) \succeq(\underbrace{w, \alpha, \ldots, \alpha}_{n \text { terms }})$, for $\alpha \geqslant \frac{1}{n} \sum_{i=1}^{n} \alpha_{i}$.

Remark 3.2 It is to be noted that Archimedean copulas with generators $\varphi(t)=2 /\left(1+e^{t}\right)$ and $\varphi(t)=(-1+\theta) /\left(-e^{t}+\theta\right)$ for $-1 \leq \theta \leq 0$ are some examples of survival copula such that $\varphi$ is log-concave, and $\frac{\varphi(1-\varphi)}{\varphi^{\prime}}$ is decreasing and convex.

The following counterexample shows that one may not get the the ordering result in Theorem 3.3 if the sufficient conditions on the generator functions are dropped.

Counterexample 3.2 Consider two series systems, each comprising of three dependent and heterogeneous components with respective hazard rate functions $r_{X_{1: 3}}(x)$ and $r_{Y_{1: 3}}(x)$, with common baseline survival function $\bar{F}(x)=e^{-(0.5 x)^{2}}, x \geq 0, \boldsymbol{\alpha}=(0.2,0.4,0.6), \boldsymbol{\beta}=(0.35,0.55,0.95)$ so that $\boldsymbol{\alpha} \succeq \boldsymbol{w}$. First we take the common generator $\varphi(x)=\log (e+x)^{-1 / a}, a=0.1$, which is not $\log$-concave but $\frac{\varphi(1-\varphi)}{\varphi^{\prime}}$ is decreasing and convex. Next we take $\varphi(x)=\left(2 /\left(1+e^{x}\right)\right)^{1 / a}$, $a=0.2$, which is log-concave but $\frac{\varphi(1-\varphi)}{\varphi^{\prime}}$ is neither decreasing nor convex. For both the cases $r_{X_{1: 3}}(x)$ and $r_{Y_{1: 3}}(x)$ are depicted in Figure 2(a) and 2(b) respectively for some finite range of $x$. From both the figures we observe that the hazard rate ordering result in Theorem 3.3 is not attained. 


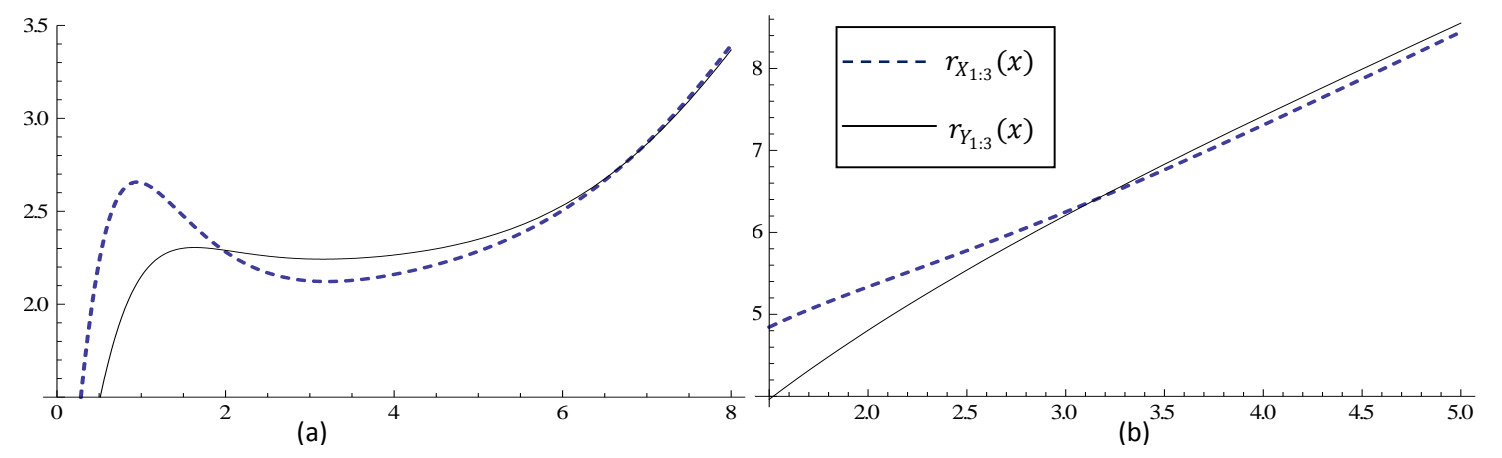

Figure 2: Plots of $r_{X_{1: 3}}(x)$ and $r_{Y_{1: 3}}(x)$ for $(\mathrm{a}) \varphi(x)$ is not log-concave, (b) $\frac{\varphi(1-\varphi)}{\varphi^{\prime}}$ is neither decreasing nor convex.

\section{Parallel systems with dependent and heterogeneous compo- nent lifetimes following PO Model}

Here, we compare the lifetimes of two parallel systems consisting of dependent and heterogeneous components having lifetimes following the PO model, with respect to some stochastic orders.

Let the lifetime vector $X=\left(X_{1}, X_{2}, \ldots, X_{n}\right)$ be a set of dependent random variables following the PO model with baseline survival function $\bar{F}$ and having the joint distribution function coupled with Archimedean survival copula with generator $\varphi$, denoted as $X \sim \operatorname{PO}(\bar{F}, \boldsymbol{\alpha}, \varphi)$, where $\boldsymbol{\alpha}=\left(\alpha_{1}, \alpha_{2}, \ldots, \alpha_{n}\right) \in \mathbb{R}_{+}^{n}$ is the proportional odds ratio vector. The distribution function of $X_{i}$ is $F_{\alpha_{i}}(x)=\frac{F(x)}{1-\bar{\alpha}_{i} \bar{F}(x)}$. The distribution function of $X_{n: n}$ is given by

$$
F_{X_{n: n}}(x)=P\left(X_{n: n} \leq x\right)=P\left(X_{i}<x, i \in I_{n}\right)=\varphi\left(\sum_{i=1}^{n} \phi\left(F_{\alpha_{i}}(x)\right)\right)=S_{2}(F(x), \boldsymbol{\alpha}, \varphi), \text { say }
$$

where $\phi(u)=\varphi^{-1}(u), u \in(0,1]$. The reversed hazard rate function of $X_{n: n}$ is obtained as

$$
\tilde{r}_{X_{n: n}}(x)=\tilde{r}(x) \frac{\varphi^{\prime}\left(\sum_{i=1}^{n} \phi\left(F_{\alpha_{i}}(x)\right)\right)}{\varphi\left(\sum_{i=1}^{n} \phi\left(F_{\alpha_{i}}(x)\right)\right)} \sum_{i=1}^{n} \phi^{\prime}\left(F_{\alpha_{i}}(x)\right) \frac{\alpha_{i} F_{\alpha_{i}}(x)}{1-\bar{\alpha}_{i} \bar{F}(x)},
$$

where $\tilde{r}$ denotes the baseline reversed hazard rate function.

Lemma 4.1 For any $x \in[0,1], S_{2}(x, \boldsymbol{\alpha}, \varphi)$ is decreasing in $\alpha_{i}, i \in I_{n}$. Furthermore $S_{2}$ is Schur-convex with respect to $\boldsymbol{\alpha}$ whenever $\varphi$ is log-concave. 
Proof: For $s \in I_{n}$,

$$
\frac{\partial S_{2}}{\partial \alpha_{s}}=-\varphi^{\prime}\left(\sum_{i=1}^{n} \phi\left(\frac{x}{1-\bar{\alpha}_{i}(1-x)}\right)\right) \phi^{\prime}\left(\frac{x}{1-\bar{\alpha}_{s}(1-x)}\right) \frac{x(1-x)}{\left(1-\bar{\alpha}_{s}(1-x)\right)^{2}}
$$

Since both $\varphi(u)$ and $\phi(u)$ are decreasing for all $u \geq 0, \frac{\partial S_{2}}{\partial \alpha_{s}} \leq 0$. So $S_{2}(x, \boldsymbol{\alpha}, \varphi)$ is decreasing in $\alpha_{i}$ for any $x \in[0,1]$.

For $s \neq t$,

$$
\begin{aligned}
& \left(\alpha_{s}-\alpha_{t}\right)\left(\frac{\partial S_{2}}{\partial \alpha_{s}}-\frac{\partial S_{2}}{\partial \alpha_{t}}\right) \\
= & -\left(\alpha_{s}-\alpha_{t}\right) \varphi^{\prime}\left(\sum_{i=1}^{n} \phi\left(v_{i}\right)\right)\left[\phi^{\prime}\left(v_{s}\right) \frac{x(1-x)}{\left(1-\bar{\alpha}_{s} x\right)^{2}}-\phi^{\prime}\left(v_{t}\right) \frac{x(1-x)}{\left(1-\bar{\alpha}_{t} x\right)^{2}}\right], v_{i}=\frac{x}{1-\bar{\alpha}_{i}(1-x)} \\
\stackrel{\text { sign }}{=} & \left(\alpha_{s}-\alpha_{t}\right)\left[-\left(-\frac{\varphi\left(\phi\left(v_{s}\right)\right)}{\varphi^{\prime}\left(\phi\left(v_{s}\right)\right)}\right) \frac{1}{1-\bar{\alpha}_{s} x}+\left(-\frac{\varphi\left(\phi\left(v_{t}\right)\right)}{\varphi^{\prime}\left(\phi\left(v_{t}\right)\right)}\right) \frac{1}{1-\bar{\alpha}_{t} x}\right] \\
\geq & 0,
\end{aligned}
$$

where the last inequality is derived using the fact that $\varphi$ is log-concave. So from Lemma 2.1. $S_{2}$ is Schur-convex in $\boldsymbol{\alpha}=\left(\alpha_{1}, \alpha_{2}, \ldots, \alpha_{n}\right)$.

Suppose there are two parallel systems with lifetime vectors $X=\left(X_{1}, X_{2}, \ldots, X_{n}\right)$ and $Y=$ $\left(Y_{1}, Y_{2}, \ldots, Y_{n}\right)$, formed out of $n$ dependent and heterogeneous components where the component lifetimes follow PO model. The following theorem compares the lifetimes of two such parallel systems in the sense of usual stochastic order.

Theorem 4.1 Suppose the lifetime vectors $X \sim P O\left(\bar{F}, \boldsymbol{\alpha}, \varphi_{1}\right)$ and $Y \sim P O\left(\bar{F}, \boldsymbol{\beta}, \varphi_{2}\right)$. If $\varphi_{1}$ or $\varphi_{2}$ is log-concave and $\phi_{1} \circ \varphi_{2}$ is superadditive, then

$$
\boldsymbol{\alpha} \succeq \boldsymbol{\beta} \text { implies } X_{n: n} \leq_{s t} Y_{n: n}
$$

Proof: If $\varphi_{1}$ is log-concave, then from Lemma 4.1 and Lemma 2.2, we have

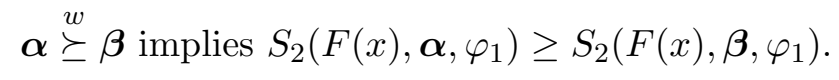

Since $\phi_{1} \circ \varphi_{2}$ is superadditive, so from Lemma 2.4 (by replacing $\varphi_{1}$ by $\varphi_{2}$ and vice versa), we have

$$
S_{2}\left(F(x), \boldsymbol{\beta}, \varphi_{1}\right) \geq S_{2}\left(F(x), \boldsymbol{\beta}, \varphi_{2}\right) .
$$

Combining (9) and (10), we get $S_{2}\left(F(x), \boldsymbol{\alpha}, \varphi_{1}\right) \geq S_{2}\left(F(x), \boldsymbol{\beta}, \varphi_{2}\right)$. That is $X_{n: n} \leq_{s t} Y_{n: n}$. 

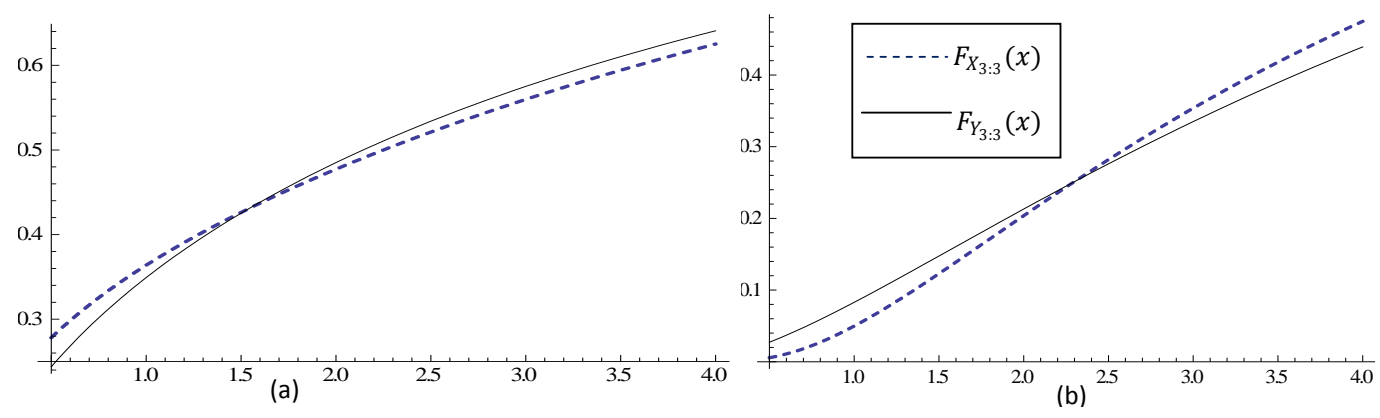

Figure 3: Plots of $F_{X_{3: 3}}(x)$ and $F_{Y_{3: 3}}(x)$ for (a) neither $\varphi_{1}$ nor $\varphi_{2}$ is log-concave, (b) $\phi_{1} \circ \varphi_{2}$ is not super additive.

Now suppose $\varphi_{2}$ is log-concave, then

$$
\begin{aligned}
S_{2}\left(F(x), \boldsymbol{\alpha}, \varphi_{1}\right) & \geq S_{2}\left(F(x), \boldsymbol{\alpha}, \varphi_{2}\right) \\
& \geq S_{2}\left(F(x), \boldsymbol{\beta}, \varphi_{2}\right)
\end{aligned}
$$

where the first inequality follows from the fact that $\phi_{1} \circ \varphi_{2}$ is superadditive, whereas the second inequality follows from the fact that $\boldsymbol{\alpha} \succeq \boldsymbol{w}$. This proves the result.

Corollary 4.1 Suppose the lifetime vectors $X \sim P O(\bar{F}, \boldsymbol{\alpha}, \varphi)$ and $Y \sim P O(\bar{F}, \boldsymbol{\beta}, \varphi)$. If $\varphi$ is log-concave, then

$$
\boldsymbol{\alpha} \succeq \boldsymbol{w} \text { implies } X_{n: n} \leq_{s t} Y_{n: n} \cdot \square
$$

The following counterexample shows that one may not get the the ordering result in Theorem 4.1 if the sufficient conditions on the generator functions are dropped.

Counterexample 4.1 Consider two parallel systems, each comprising of three dependent and heterogeneous components with respective distribution functions $F_{X_{3: 3}}(x)=S_{2}\left(F(x), \boldsymbol{\alpha}, \varphi_{1}\right)$ and $F_{Y_{3: 3}}(x)=S_{2}\left(F(x), \boldsymbol{\beta}, \varphi_{2}\right)$, where $F(x)=1-e^{-x^{0.5}}, x \geq 0, \boldsymbol{\alpha}=(0.9,1.45,2.15), \boldsymbol{\beta}=$ $(1.2,1.95,2.65)$ so that $\boldsymbol{\alpha} \succeq \boldsymbol{w}$. First we take $\varphi_{1}(x)=\theta_{1} / \log \left(x+e^{\theta_{1}}\right)$ and $\varphi_{2}(x)=e^{1-(1+x)^{1 / \theta_{2}}}$ with $\theta_{1}=0.9$ and $\theta_{2}=8$ so that neither $\varphi_{1}$ nor $\varphi_{2}$ is log-concave but $\phi_{1} \circ \varphi_{2}$ is super additive. Next we take $\varphi_{1}(x)=e^{\left(1-e^{x}\right) / \theta_{1}}$ and $\varphi_{2}(x)=\left(2 /\left(e^{x}+1\right)\right)^{1 / \theta_{2}}$ with $\theta_{1}=0.9$ and $\theta_{2}=0.2$ so that $\varphi_{1}$ is log-concave but $\phi_{1} \circ \varphi_{2}$ is not super additive. For both the cases $F_{X_{3: 3}}(x)$ and $F_{Y_{3: 3}}(x)$ are depicted in Figure 3(a) and 3(b) respectively for some finite range of $x$. From both the figures we observe that the stochastic ordering result in Theorem 4.1 is not attained.

Next we show the reversed hazard rate order of lifetimes of two parallel systems of dependent and heterogeneous components. 
Theorem 4.2 Suppose the lifetime vectors $X \sim P O(\bar{F}, \boldsymbol{\alpha}, \varphi)$ and $Y \sim P O(\bar{F}, \boldsymbol{\beta}, \varphi)$. If $\varphi$ is log-concave and $\frac{\varphi(1-\varphi)}{\varphi^{\prime}}$ is decreasing and convex, then

$$
\boldsymbol{\alpha} \succeq \boldsymbol{\beta} \text { implies } X_{n: n} \leq_{r h r} Y_{n: n}
$$

Proof: From (8), the reversed hazard function of $X_{n: n}$ is given by

$$
\begin{aligned}
\tilde{r}_{X_{n: n}}(x) & =\frac{\tilde{r}(x)}{\bar{F}(x)} \frac{\varphi^{\prime}\left(\sum_{i=1}^{n} \phi\left(F_{\alpha_{i}}(x)\right)\right)}{\varphi\left(\sum_{i=1}^{n} \phi\left(F_{\alpha_{i}}(x)\right)\right)} \sum_{i=1}^{n} \frac{F_{\alpha_{i}}(x)}{\varphi^{\prime}\left(\phi\left(F_{\alpha_{i}}(x)\right)\right)} \bar{F}_{\alpha_{i}}(x) \\
& =\frac{\tilde{r}(x)}{\bar{F}(x)} \frac{\varphi^{\prime}\left(\sum_{i=1}^{n} \phi\left(F_{\alpha_{i}}(x)\right)\right)}{\varphi\left(\sum_{i=1}^{n} \phi\left(F_{\alpha_{i}}(x)\right)\right)} \sum_{i=1}^{n} \frac{\varphi\left(\phi\left(F_{\alpha_{i}}(x)\right)\right)}{\varphi^{\prime}\left(\phi\left(F_{\alpha_{i}}(x)\right)\right)}\left(1-\varphi\left(\phi\left(F_{\alpha_{i}}(x)\right)\right)\right) \\
& =\frac{\tilde{r}(x)}{\bar{F}(x)} \frac{\varphi^{\prime}\left(\sum_{i=1}^{n} \xi_{i}\right)}{\varphi\left(\sum_{i=1}^{n} \xi_{i}\right)} \sum_{i=1}^{n} \frac{\varphi\left(\xi_{i}\right)}{\varphi^{\prime}\left(\xi_{i}\right)}\left(1-\varphi\left(\xi_{i}\right)\right)
\end{aligned}
$$

where $\xi_{i}=\phi\left(F_{\alpha_{i}}(x)\right)$. Now, for $s \in I_{n}$,

$$
\begin{aligned}
\frac{\tilde{r}_{X_{n: n}}(x)}{\partial \alpha_{s}}= & \frac{\tilde{r}(x)}{\bar{F}(x)}\left[\frac{\partial}{\partial \xi_{s}}\left(\frac{\varphi^{\prime}\left(\sum_{i=1}^{n} \xi_{i}\right)}{\varphi\left(\sum_{i=1}^{n} \xi_{i}\right)}\right) \frac{\partial \xi_{s}}{\partial \alpha_{s}} \sum_{i=1}^{n} \frac{\varphi\left(\xi_{i}\right)\left(1-\varphi\left(\xi_{i}\right)\right)}{\varphi^{\prime}\left(\xi_{i}\right)}+\right. \\
& \left.\frac{\varphi^{\prime}\left(\sum_{i=1}^{n} \xi_{i}\right)}{\varphi\left(\sum_{i=1}^{n} \xi_{i}\right)} \frac{\partial}{\partial \xi_{s}}\left(\frac{\varphi\left(\xi_{s}\right)\left(1-\varphi\left(\xi_{s}\right)\right)}{\varphi^{\prime}\left(\xi_{s}\right)}\right) \frac{\partial \xi_{s}}{\partial \alpha_{s}}\right] .
\end{aligned}
$$

Note that $\xi_{s}$ is increasing in $\alpha_{s}$ and $\frac{\partial \xi_{s}}{\partial \alpha_{s}}$ is decreasing in $\alpha_{s}$. Since $\varphi$ is log-concave and $\frac{\varphi(1-\varphi)}{\varphi^{\prime}}$ is decreasing, we have $\frac{\tilde{r}_{X_{n: n}}(x)}{\partial \alpha_{s}} \geq 0$. Again

$$
\frac{\partial}{\partial \xi_{s}}\left(\frac{\varphi^{\prime}\left(\sum_{i=1}^{n} \xi_{i}\right)}{\varphi\left(\sum_{i=1}^{n} \xi_{i}\right)}\right)=\frac{\partial}{\partial \xi_{t}}\left(\frac{\varphi^{\prime}\left(\sum_{i=1}^{n} \xi_{i}\right)}{\varphi\left(\sum_{i=1}^{n} \xi_{i}\right)}\right) \text {, for } s \neq t \text {. }
$$

For $s \neq t$,

$$
\begin{array}{ll} 
& \left(\alpha_{s}-\alpha_{t}\right)\left(\frac{\tilde{r}_{X_{n: n}}}{\partial \alpha_{s}}-\frac{\tilde{r}_{X_{n: n}}}{\partial \alpha_{t}}\right) \\
\stackrel{\text { sign }}{=}\left(\alpha_{s}-\alpha_{t}\right)\left(\frac{\partial \xi_{s}}{\partial \alpha_{s}}-\frac{\partial \xi_{t}}{\partial \alpha_{t}}\right)+\left(\alpha_{s}-\alpha_{t}\right)\left(-\frac{\varphi^{\prime}\left(\sum_{i=1}^{n} \xi_{i}\right)}{\varphi\left(\sum_{i=1}^{n} \xi_{i}\right)}\right) \times \\
& {\left[\left(-\frac{\partial}{\partial \xi_{s}}\left(\frac{\varphi\left(\xi_{s}\right)\left(1-\varphi\left(\xi_{s}\right)\right)}{\varphi^{\prime}\left(\xi_{s}\right)}\right)\right) \frac{\partial \xi_{s}}{\partial \alpha_{s}}-\left(-\frac{\partial}{\partial \xi_{t}}\left(\frac{\varphi\left(\xi_{t}\right)\left(1-\varphi\left(\xi_{t}\right)\right)}{\varphi^{\prime}\left(\xi_{t}\right)}\right)\right) \frac{\partial \xi_{t}}{\partial \alpha_{t}}\right]} \\
\leq & 0,
\end{array}
$$

as $\frac{\varphi(1-\varphi)}{\varphi^{\prime}}$ is decreasing and convex. Thus we have $\tilde{r}_{X_{n: n}}(x)$ is increasing in $\alpha_{i}, i \in I_{n}$ and Schur-concave in $\boldsymbol{\alpha}=\left(\alpha_{1}, \alpha_{2}, \ldots, \alpha_{n}\right)$. Then from Lemma 2.2, we have

$$
\boldsymbol{\alpha} \succeq \boldsymbol{\beta} \text { implies } \tilde{r}_{X_{n: n}}(x) \leq \tilde{r}_{Y_{n: n}}(x)
$$




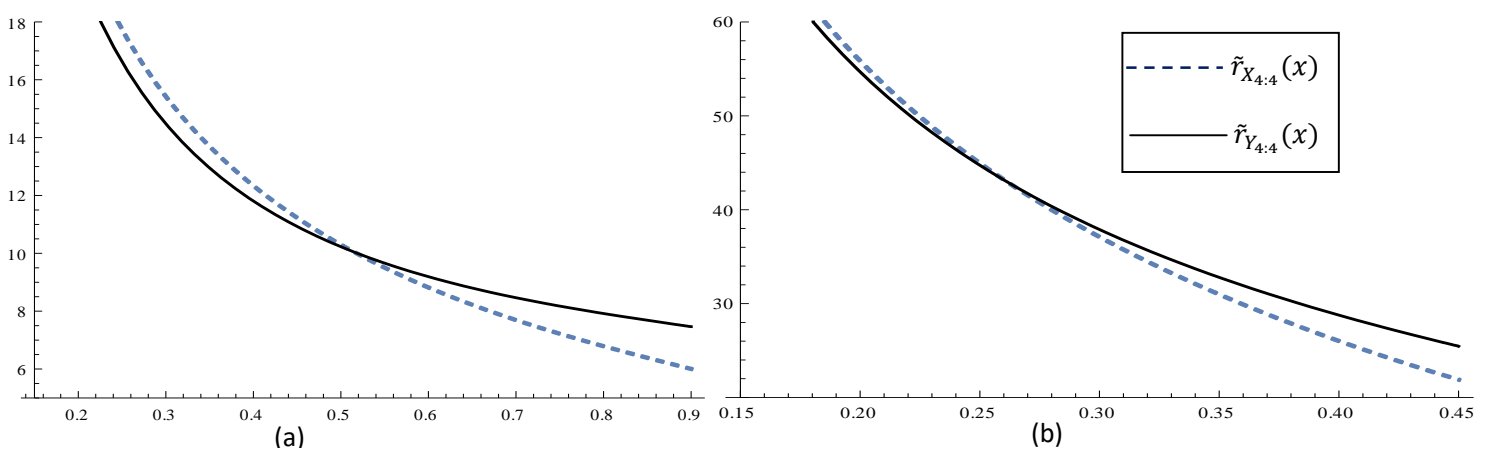

Figure 4: Plots of $\tilde{r}_{X_{4: 4}}(x)$ and $\tilde{r}_{Y_{4: 4}}(x)$ for $(\mathrm{a}) \varphi(x)$ is not log-concave, (b) $\frac{\varphi(1-\varphi)}{\varphi^{\prime}}$ is neither decreasing nor convex.

Hence the theorem follows. The following counterexample shows that one may not get the the ordering result in Theorem 4.2 if the sufficient conditions on the generator functions are dropped.

Counterexample 4.2 Consider two parallel systems, each comprising of four dependent and heterogeneous components with respective reversed hazard rate functions $\tilde{r}_{X_{4: 4}}(x)$ and $\tilde{r}_{Y_{4: 4}}(x)$, with common baseline survival function $\bar{F}(x)=e^{-x^{3}}, x \geq 0, \boldsymbol{\alpha}=(0.2,0.6,1.5,3.5), \boldsymbol{\beta}=$ $(0.8,0.9,4.5,5.5)$ so that $\boldsymbol{\alpha} \succeq \boldsymbol{w}$. First we take the common generator $\varphi(x)=(1 /(a x+1))^{1 / a}$, $a=0.2$, which is not log-concave but $\frac{\varphi(1-\varphi)}{\varphi^{\prime}}$ is decreasing and convex. Next we take $\varphi(x)=$ $\left(2 /\left(1+e^{x}\right)\right)^{1 / a}, a=0.2$, which is log-concave but $\frac{\varphi(1-\varphi)}{\varphi^{\prime}}$ is neither decreasing nor convex. For both the cases $\tilde{r}_{X_{4: 4}}(x)$ and $\tilde{r}_{Y_{4: 4}}(x)$ are depicted in Figure 4 (a) and 4 (b) respectively for some finite range of $x$. From both the figures we observe that the reversed hazard rate ordering result in Theorem 4.2 is not attained.

\section{Applications}

In this section, we highlight some potential applications of the established results. Consider a series (or a parallel) system of $n$ components having dependent lifetimes. It is quite practical that the odds functions of all the components (i.e. the odds of surviving beyond a specified time $t$ ) may not be the same for various possible reasons, like the components are manufactured by different manufacturing units or they are subjected to different levels of stress. So let the odds function of $i$ th component is proportional to a baseline odds function with proportionality constant (odds ratio) $\alpha_{i}, i=1,2, \ldots, n$. Now consider another series (or parallel) system of $n$ dependent components having different odds ratios $\beta_{i}, i=1,2, \ldots, n$. Even if a same system operates in two different levels of environments/stress (e.g., voltage, temperature, compression and tension), then reliability characteristics (e.g., odds function) of a component of the system 
generally will not be the same in the two different environments. So it is a subject of interest to compare lifetimes of two such systems, i.e. under what conditions one system will be more reliable than other. Theorems 3.1 and 3.2 (resp. Theorem 4.1) give the conditions on the corresponding odds ratio vectors and the generators of the survival copulas under which a series (resp. parallel) system will have stochastically longer lifetime than that of the other. Similarly Theorem 3.3 (resp. Theorem 4.2 ) gives the conditions under which failure rate of a series (resp. parallel) system will be smaller than that of the other.

Next we show that using our proposed results one can compare the lifetime of two series systems whose components are subjected to random shock instantaneously [8]. Suppose random variable $X_{i}$ denotes the lifetime of $i$-th component of the series system. Define Bernoulli random variable $I_{p_{i}}$ associated with $X_{i}$, where $I_{p_{i}}=1$ if shock does not occur and 0 if shock occurs with $p_{i}=P\left(I_{p_{i}}=1\right), i=1, \ldots, n$. Assume that $I_{p_{1}}, \ldots, I_{p_{n}}$ are independent random variables, and also they are independent of $X_{1}, \ldots, X_{n}$. Let $X_{i}^{*}=X_{i} I_{p_{i}}, i=1, \ldots, n$, and denote $X_{1: n}^{*}=\min \left(X_{1}^{*}, \ldots, X_{n}^{*}\right)$. Similarly assume that $I_{q_{1}}, \ldots, I_{q_{n}}$ are independent Bernoulli random variables, and also they are independent of $Y_{i}$ 's with $q_{i}=P\left(I_{q_{i}}=1\right), i=1, \ldots, n$. Denote $Y_{1: n}^{*}=\min \left(Y_{1}^{*}, \ldots, Y_{n}^{*}\right)$, where $Y_{i}^{*}=Y_{i} I_{q_{i}}, i=1, \ldots, n$. Here $X_{1: n}^{*}$ represents the lifetime of a series system whose components are subjected to random shock instantaneously. Similarly $Y_{1: n}^{*}$ represents the the lifetime of another such series system. Now, if $X \sim P O\left(\bar{F}, \boldsymbol{\alpha}, \varphi_{1}\right)$ and $Y \sim P O\left(\bar{F}, \boldsymbol{\beta}, \varphi_{2}\right)$, then with the help of the Theorems 3.1, 3.2, 3.3, and the associated corollaries 3.1, 3.2, we can establish following stochastic comparisons between such smallest order statistics from the fact that $P\left(X_{1: n}^{*}>x\right)=P\left(X_{1}>x, \ldots, X_{n}>x\right) P\left(I_{p_{i}}=1, i \in I_{n}\right)=$ $P\left(X_{1: n}>x\right) \prod_{i}^{n} p_{i}$.

Theorem 5.1 Suppose the lifetime vectors $X \sim P O\left(\bar{F}, \boldsymbol{\alpha}, \varphi_{1}\right)$ and $Y \sim P O\left(\bar{F}, \boldsymbol{\beta}, \varphi_{2}\right)$. If $\varphi_{1}$ or $\varphi_{2}$ is log-convex, $\phi_{2} \circ \varphi_{1}$ is superadditive and $\prod_{i}^{n} p_{i} \leq \prod_{i}^{n} q_{i}$, then

$$
\boldsymbol{\alpha} \succeq \boldsymbol{p} \text { implies } X_{1: n}^{*} \leq_{s t} Y_{1: n}^{*}
$$

Corollary 5.1 Suppose the lifetime vectors $X \sim P O(\bar{F}, \boldsymbol{\alpha}, \varphi)$ and $Y \sim P O(\bar{F}, \boldsymbol{\beta}, \varphi)$. If $\varphi$ is log-convex and $\prod_{i}^{n} p_{i} \leq \prod_{i}^{n} q_{i}$, Then

$$
\boldsymbol{\alpha} \succeq \boldsymbol{\beta} \text { implies } X_{1: n}^{*} \leq_{s t} Y_{1: n}^{*}
$$

Theorem 5.2 Suppose the lifetime vectors $X \sim P O\left(\bar{F}, \boldsymbol{\alpha}, \varphi_{1}\right)$ and $Y \sim P O\left(\bar{F}, \boldsymbol{\beta}, \varphi_{2}\right)$. If $\phi_{2} \circ \varphi_{1}$ is superadditive and $\prod_{i}^{n} p_{i} \leq \prod_{i}^{n} q_{i}$, then

$$
\boldsymbol{\alpha} \succeq \boldsymbol{\beta} \text { implies } X_{1: n}^{*} \leq_{s t} Y_{1: n}^{*}
$$

Corollary 5.2 Suppose the lifetime vectors $X \sim P O(\bar{F}, \boldsymbol{\alpha}, \varphi)$ and $Y \sim P O(\bar{F}, \boldsymbol{\beta}, \varphi)$. If 
$\prod_{i}^{n} p_{i} \leq \prod_{i}^{n} q_{i}$, then

$$
\boldsymbol{\alpha} \stackrel{w}{\succeq} \boldsymbol{\beta} \text { implies } X_{1: n}^{*} \leq_{s t} Y_{1: n}^{*} .
$$

Theorem 5.3 Suppose the lifetime vectors $X \sim P O(\bar{F}, \boldsymbol{\alpha}, \varphi)$ and $Y \sim P O(\bar{F}, \boldsymbol{\beta}, \varphi)$. If $\varphi$ is log-concave and $\frac{\varphi(1-\varphi)}{\varphi^{\prime}}$ is decreasing and concave, then

$$
\boldsymbol{\alpha} \stackrel{w}{\succeq} \text { implies } X_{1: n}^{*} \leq_{h r} Y_{1: n}^{*} .
$$

We will end this section by mentioning an other potential application. In actuarial science, $X_{1: n}^{*}$ corresponds to the smallest claim amount in a portfolio of risks [1, 17, 24, where $X_{i}$ 's represent sizes of random claims of multiple risks covered by a policy that can be made in an insurance period and the corresponding $I_{p_{i}}$ 's indicate the occurrence of these claims. That means $I_{p_{i}}=1$ whenever the $i$ th policy makes random claim $X_{i}$ and $I_{p_{i}}=0$ whenever there is no claim. Similarly suppose $Y_{1: n}^{*}$ represents the smallest claim amount in an another portfolio of risks. The above theorems can be used in stochastic comparisons between the smallest claim amounts of two different portfolio of risks.

\section{Acknowledgements:}

The authors are thankful to the Area Editor and the anonymous Reviewers for the constructive comments and suggestions, which lead to an improved version of the manuscript.

\section{References}

\section{References}

[1] G. Barmalzan, A.T.P. Najafabadi, N. Balakrishnan, Ordering properties of the smallest and largest claim amounts in a general scale model, Scandinavian Actuarial Journal 2017 (2017) 105-124.

[2] S. Bennett, Analysis of survival data by the proportional odds model, Statistics in Medicine 2 (1983) 273-277.

[3] J.L. Bon, E. Păltănea, Ordering properties of convolutions of exponential random variables, Lifetime Data Analysis 5 (1999) 185-192.

[4] D. Collett, Modelling survival data in medical research, 2nd edition, Chapman and Hall/CRC, 2004.

[5] G.M. Cordeiro, A.J. Lemonte, E.M.M. Ortega, The Marshall-Olkin family of distributions: mathematical properties and new models, Journal of Statistical Theory and Practice 8(2) (2014) 343-366.

[6] W. Ding, Y. Zhang, Relative ageing of series and parallel systems: Effects of dependence and heterogeneity among components, Operations Research Letters 46 (2018) 219-224. 
[7] L. Fang, N. Balakrishnan N, Ordering results for the smallest and largest order statistics from independent heterogeneous exponential-Weibull random variables, Statistics 50 (2016) 1195-1205.

[8] L. Fang, N. Balakrishnan, Ordering properties of the smallest order statistics from generalized Birnbaum-Saunders models with associated random shocks, Metrika 81(1) (2018) 19-35.

[9] R. Fang, C. Li, X. Li, Stochastic comparisons on sample extremes of dependent and heterogenous observations, Statistics 50 (2016) 930-955.

[10] N. Gupta, L.K. Patra, S. Kumar, S, Stochastic comparisons in systems with Frèchet distributed components, Operations Research Letters 43 (2015) 612-615.

[11] N.K. Hazra, M.R. Kuiti, M. Finkelstein, A.K. Nanda, On stochastic comparisons of maximum order statistics from the Location-Scale family of distributions, Journal of Multivariate Analysis 160 (2017) 31-41.

[12] S.N.U.A. Kirmani, R.C. Gupta, On the proportional odds model in survival analysis, Annals of the Institute of Statistical Mathematics 53(2) (2001) 203-216.

[13] B. Khaledi, S.C. Kochar, Dispersive ordering among linear combinations of uniform random variables, Journal of Statistical Planning and Inference 100 (2002) 13-21.

[14] P. Kundu, A.K. Nanda, Reliability study of proportional odds family of discrete distributions, Communications in Statistics - Theory and Methods 47(5) (2018) 1091-1103.

[15] P. Kundu, N.K. Hazra, A.K. Nanda, Reliability study of series and parallel systems of heterogeneous component lifetimes following proportional odds model, Statistics (2020). DOI: 10.1080/02331888.2020.1722670.

[16] X. Li, R. Fang, Ordering properties of order statistics from random variables of Archimedean copulas with applications, Journal of Multivariate Analysis 133 (2015) 304-320.

[17] C. Li, X. Li, Hazard rate and reversed hazard rate orders on extremes of heterogeneous and dependent random variables, Statistics and Probability Letters 146 (2019) 104-111.

[18] A.W. Marshall, I. Olkin, A new method of adding a parameter to a family of distributions with applications to the exponential and Weibull families, Biometrika 84 (1997) 641-652.

[19] A.W. Marshall, I. Olkin, B.C. Arnold, Inequalities: Theory of Majorization and its Applications, Springer, New York, 2011.

[20] A.K. Nanda, S. Das, Stochastic orders of the Marshall-Olkin extended distribution, Statistics and Probability Letters 82 (2012) 295-302.

[21] J. Navarro, F. Spizzichino, Comparisons of series and parallel systems with components sharing the same copula, Applied Stochastic Models in Business and Industries 26 (2010) 775-791.

[22] A.J. Rossini, A.A. Tsiatis, A Semiparametric proportional odds regression model for the analysis of current status data, Journal of the American Statistical Association 91(434) (1996) 713-721. 
[23] M. Shaked, J.G. Shanthikumar, Stochastic Orders, Springer-Verlag, New York, 2007.

[24] Y. Zhang, X. Cai, P. Zhao, Ordering properties of extreme claim amounts from heterogeneous portfolios, ASTIN Bulletin: The Journal of the IAA 49(2) (2019) 525-554.

[25] W. Zhang, Z. Zhang, X. Li, Q. Li, Fitting proportional odds model to case-control data with incorporating Hardy-Weinberg Equilibrium, Scientific Reports 5, Article number: 17286 (2015). 